\title{
Tunneling Anisotropic Magnetoresistance and Spin-Orbit Coupling in Fe/GaAs/Au Tunnel Junctions
}

\author{
J. Moser, A. Matos-Abiague, D. Schuh, W. Wegscheider, J. Fabian, and D. Weiss \\ Institut für Experimentelle und Angewandte Physik, Universität Regensburg, 93040 Regensburg, Germany
}

(Received 13 November 2006; published 2 August 2007)

\begin{abstract}
We report the observation of tunneling anisotropic magnetoresistance effect in the epitaxial metalsemiconductor system $\mathrm{Fe} / \mathrm{GaAs} / \mathrm{Au}$. The observed twofold anisotropy of the resistance can be switched by reversing the bias voltage, suggesting that the effect originates from the interference of the spin-orbit coupling at the interfaces. Corresponding model calculations reproduce the experimental findings very well.
\end{abstract}

DOI: 10.1103/PhysRevLett.99.056601

PACS numbers: 72.25.Dc, 75.47. $-\mathrm{m}$

Tunneling magnetoresistance (TMR) devices consist of a tunneling barrier, sandwiched between two ferromagnetic layers. Such systems find widespread use in sensor and memory applications as they exhibit a large resistance difference for parallel and antiparallel alignments of the ferromagnets' magnetization [1]. The TMR effect relies, within the simplest model [2], on the different spin polarizations at the Fermi energy $E_{F}$ in the ferromagnets; it is absent if one ferromagnetic layer is replaced by a normal metal. Hence, it came as a surprise that a spin-valve-like tunnel magnetoresistance was found in ( $\mathrm{Ga}, \mathrm{Mn}) \mathrm{As} /$ alumina/Au sandwiches [3]. The origin of the effect, labeled tunneling anisotropic magnetoresistance (TAMR) $[3,4]$, was associated with the anisotropic density of states in ferromagnetic ( $\mathrm{Ga}, \mathrm{Mn})$ As. An enhanced anisotropic magnetoresistance (AMR) effect measured across a constriction in a $(\mathrm{Ga}, \mathrm{Mn}) \mathrm{As}$ film was ascribed to the TAMR effect, too [5]. In both experiments, the fourfold symmetry, expected if the $(\mathrm{Ga}, \mathrm{Mn})$ As hole density of states is involved, was broken and (partially) ascribed to strain in (Ga, Mn)As.

Here we show that a TAMR effect can also be observed in sandwiches involving a conventional ferromagnet such as iron. A stack of Fe, GaAs, and Au, with iron grown epitaxially on the GaAs tunneling barrier, shows a uniaxial anisotropy of the tunneling magnetoresistance. Depending on the bias voltage, the high resistance state is either observed for the magnetization $\mathbf{M}$ oriented in the [110] or the [110] direction. We propose a theoretical model in which the $C_{2 v}$ symmetry, resulting from the interference of Bychkov-Rashba and Dresselhaus spin-orbit interactions, affects the tunneling probability, giving rise to the observed twofold symmetry.

A sketch of the system is shown in Fig. 1(a). The $13 \mathrm{~nm}$ thick epitaxial iron layer was grown on an $8 \mathrm{~nm}$ thin GaAs (001) barrier by transferring the freshly grown GaAs heterojunction from the molecular beam epitaxy chamber to a magnetron sputtering system without breaking the ultrahigh vacuum (UHV). The quality of the interface of a sample from the same wafer was checked by highresolution transmission electron microscopy [6]. The Fe layer was covered by $50 \mathrm{~nm} \mathrm{Co} \mathrm{[7]} \mathrm{and} 100 \mathrm{~nm} \mathrm{Au}$, forming the back contact. The wafer was then glued upside down to another substrate, and the original substrate was etched away. Finally, the circular, $150 \mathrm{~nm}$ thick top Au contact was made by optical lithography, selective etching of AlGaAs, and UHV magnetron sputtering. At the

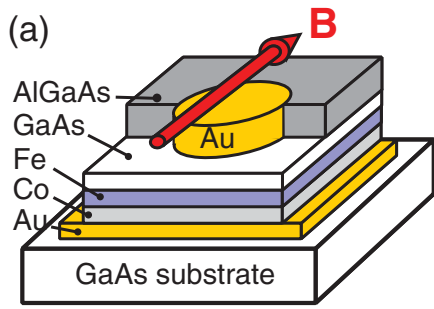

(b)
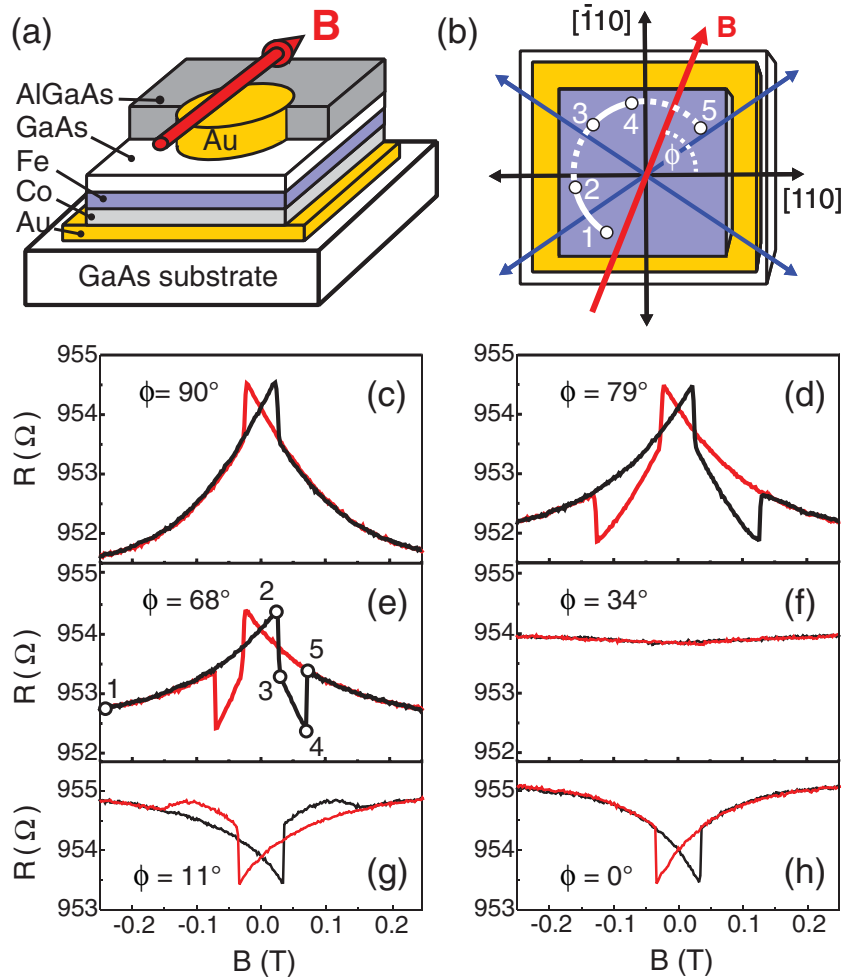

FIG. 1 (color). (a) Schematic picture of a Fe/GaAs/Au tunnel structure; the top $\mathrm{Au}$ contact has a diameter of $16 \mu \mathrm{m}$; the square bottom Fe contact has a side length of $100 \mu \mathrm{m}$ and is attached to Fe leads; (b) schematic top view on the iron layer; (c)-(h) tunneling resistance depending on $\mathbf{B}$ swept under different angles $\phi$ measured at $4.2 \mathrm{~K}$ and $-90 \mathrm{mV}$ bias (Au contact grounded). The double step switching mechanism is illustrated in (b) and (e) (see text). Data taken between $\phi=90^{\circ}$ and $180^{\circ}$ show the same characteristics. 
$\mathrm{Fe} / \mathrm{GaAs}$ and the $\mathrm{Au} / \mathrm{GaAs}$ interface, Schottky barriers form. Though the barrier heights on both sides are expected to be slightly different, they have a typical height of $\sim 0.75 \mathrm{eV}$. Hence, the GaAs layer constitutes a nearly rectangular tunneling barrier for electrons. Tunneling in such systems was studied previously [8], and a TMR effect has been observed $[6,8]$. In total, four batches of samples which differ in the preparation of the Au layer (see, e.g., Ref. [6]) or in an additional annealing step $\left(150{ }^{\circ} \mathrm{C}\right.$ for $\left.1 \mathrm{~h}\right)$ were investigated. As the described features are essentially independent of these details, we focus on the results of one sample below.

The measurements were carried out at $4.2 \mathrm{~K}$ using a superconducting coil to generate the external magnetic field $\mathbf{B}$. We used an HP semiconductor parameter analyzer 4155A to probe the resistance drop across the GaAs barrier in a four-point configuration with the top $\mathrm{Au}$ contact grounded. To vary the direction $\mathbf{M}$ of iron, the sample was mounted in a rotatable sample holder enabling a $360^{\circ}$ in-plane rotation of $\mathbf{B}$. The direction of $\mathbf{B}$ is given by its angle with respect to the GaAs [110] direction. The $I-V$ characteristics, measured between the $\mathrm{Au}$ and $\mathrm{Fe}$ contact, are strongly nonlinear (not shown). This suggests that electron transport is, as in previous TMR experiments, dominated by quantum mechanical tunneling [8].

Our Letter is about the anisotropy of the tunneling resistance with respect to the in-plane magnetization $\mathbf{M}$ of the Fe contact. Epitaxial $\mathrm{Fe}$ has both a cubic bulk anisotropy as well as an uniaxial contribution from the interface. Magnetization reversal for an in-plane B field takes place in two steps explained by nucleation and propagation of $90^{\circ}$ domain walls [9]. Figures 1(c)-1(h) display the tunneling resistance as a function of magnetic field $\mathbf{B}$ swept in different directions. Figure 1(c) shows the resistance for B swept at an angle of $\phi=90^{\circ}$ ([1110] direction) from negative saturation to positive saturation and back. The figure focuses on the interesting region between -0.25 and $+0.25 \mathrm{~T}$. A clear spin-valve-like signal characterized by one switching event (one jump in $R$ ) is observed if $\mathbf{B}$ is applied along this hard direction. If $\mathbf{B}$ is applied $11^{\circ}$ off the hard [110] axis, the second switching process occurs at $\sim 0.12 \mathrm{~T}$, manifested in Fig. 1(d). Decreasing $\phi$, the second switching point is shifted towards smaller $B$ [Fig. 1(e)]. This two-step switching process is described in more detail for $\phi=68^{\circ}$ in Fig. 1(e). Starting close to saturation at $-\mathbf{B}$ [point 1 in Fig. 1(b)], $\mathbf{M}$

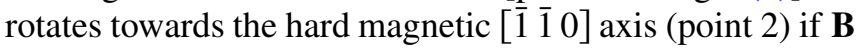
is reversed and increased towards positive field values. First, $\mathbf{M}$ switches from near the easy axis closest to the original direction of $\mathbf{B}$ beyond the easy axis located $\sim 90^{\circ}$ sideways from this one (point 3). Increasing $\mathbf{B}$ further drives $\mathbf{M}$ towards the [1110] direction (point 4) until, in the second switching event, $\mathbf{M}$ jumps near the easy direction closest to the new $\mathbf{B}$ direction (point 5). The signal disappears if $\mathbf{B}$ is swept along an easy direction-in the present sample lying at $\phi=34^{\circ}$ [Fig. 1(f)] - and changes sign for $\mathbf{B}$ close to [Fig. 1(g)] or along the hard [110] direction [Fig. 1(h)].

Though reminiscent of the AMR effect, our results cannot be explained by the AMR effect of the iron layer. The resistance change caused by the AMR effect of the Fe layer of only $\sim 4 \mathrm{~m} \Omega$ is much smaller than the observed change in the tunneling resistance of about $3.5 \Omega$.

The symmetry of the TAMR becomes more explicit at higher $\mathbf{B}$, where $\mathbf{M}$ is forced to follow the direction of the external field. The data displayed in the polar plot in Fig. 2(a) are normalized to the resistance in the hard [110] direction. An uniaxial anisotropy evincing the shape of a "horizontal 8" is clearly manifested. The resistance in the [110] direction is typically $\sim 0.4 \%$ smaller than in the [110] direction. This anisotropy explains the resistance

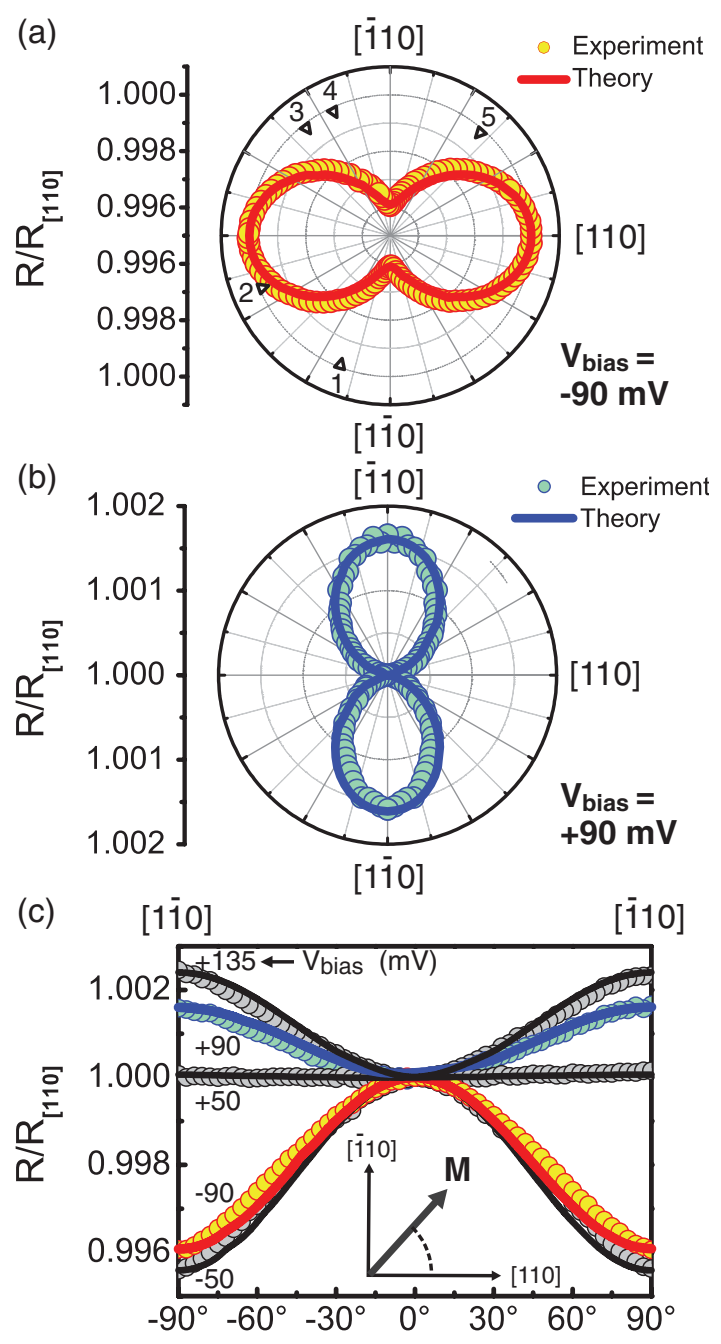

FIG. 2 (color). (a) $\phi$ scan of the tunneling resistance at $4.2 \mathrm{~K}$ and $-90 \mathrm{mV}$ bias in a saturation magnetic field $|\mathbf{B}|=0.5 \mathrm{~T}$ and a theoretical fit; (b) $\phi$ scan at $+90 \mathrm{mV}$; (c) $\phi$ scans for different bias voltages. Symbols correspond to experimental results for $-90,-50,50,90$, and $135 \mathrm{mV}$ bias; solid lines correspond to theoretical results with $\alpha_{l}=42.3 \mathrm{eV} \AA^{2}, \alpha_{l}=45.8 \mathrm{eV} \AA^{2}$, $\alpha_{l}=-0.6 \mathrm{eV} \AA^{2}, \alpha_{l}=-17.4 \mathrm{eV} \AA^{2}$, and $\alpha_{l}=-25.1 \mathrm{eV} \AA^{2}$, respectively. The absolute resistance values range between $750 \Omega$ and $974 \Omega$ in the investigated voltage regime. 
jumps observed in Figs. 1(c)-1(h): The actual direction of $\mathbf{M}$ determines R. These directions highlighted by triangles in Fig. 2(a) correspond to the directions, taken up by the magnetization $\mathbf{M}$ in Fig. 1(e) for the marked $\mathbf{B}$ values. The thin red line is the result of a model discussed below.

The anisotropy depends on the bias voltage. If the bias is reversed from -90 to $+90 \mathrm{mV}$, the " 8 " is rotated by $90^{\circ}$, shown in Fig. 2(b). The bias dependencies of the resistances' angular characteristics are summarized in Fig. 2(c). While for $V>50 \mathrm{mV}, R$ is larger for the [110] directions, for $V<50 \mathrm{mV}, R$ is largest for the [110] directions. Similar behavior was found for all samples investigated. Preliminary experiments also show that the anisotropy is reduced by only $\sim 25 \%$ at $100 \mathrm{~K}$.

We propose here that the twofold symmetry of the TAMR is a consequence of the anisotropic spin-orbit interaction (SOI). Indeed, the combination of bulk inversion asymmetry (Dresselhaus SOI) [10-12] and structure inversion asymmetry (Bychkov-Rashba SOI) [12] in GaAslike heterostructures leads to a SOI with $C_{2 v}$ symmetry, which is the symmetry of the GaAs interface with both $\mathrm{Fe}$ and $\mathrm{Au}$ layers. Based on this observation, we consider the following model Hamiltonian for describing the tunneling across our metal/semiconductor heterojunction: $H=$ $H_{0}+H_{Z}+H_{\mathrm{BR}}+H_{D}$. Here

$$
H_{0}=-\frac{\hbar^{2}}{2} \nabla\left[\frac{1}{m(z)} \nabla\right]+V_{z}
$$

with $m(z)$ the electron effective mass [in terms of the bare electron mass $m_{0}$, we assume $m=m_{c}=0.067 m_{0}$ in the central (GaAs) region and $m=m_{l}=m_{r} \approx m_{0}$ in the left $(\mathrm{Fe})$ and right $(\mathrm{Au})$ regions] and $V(z)$ the conduction band profile along the growth direction $z$ of the heterostructure [see Fig. 3(a)].

The Zeeman spin splitting due to both the exchange field (in the $\mathrm{Fe}$ region) and the external magnetic field in the $\mathrm{Fe}$ and $\mathrm{Au}$ [13] is given by

$$
H_{Z}=-\frac{\Delta(z)}{2} \mathbf{n} \cdot \boldsymbol{\sigma}
$$

Here $\Delta(z)$ represents the Zeeman energy in the different regions, $\boldsymbol{\sigma}$ is a vector whose components are the Pauli matrices, and $\mathbf{n}$ is a unit vector defining the spin quantization axis determined by the in-plane magnetization direction in $\mathrm{Fe}$.

The Bychkov-Rashba SOI due to the structure inversion asymmetry at the interfaces can be written as [14]

$$
H_{\mathrm{BR}}=\frac{1}{\hbar} \sum_{i=l, r} \alpha_{i}\left(\sigma_{x} p_{y}-\sigma_{y} p_{x}\right) \delta\left(z-z_{i}\right),
$$

where $\alpha_{l}\left(\alpha_{r}\right)$ denotes the SOI strength at the left (right) interface $z_{l}=0\left(z_{r}=d\right)$. We note that, inside the GaAs barrier, there is also a Bychkov-Rashba SOI contribution induced by the applied bias. However, this contribution is negligible for our system, and we neglect it.

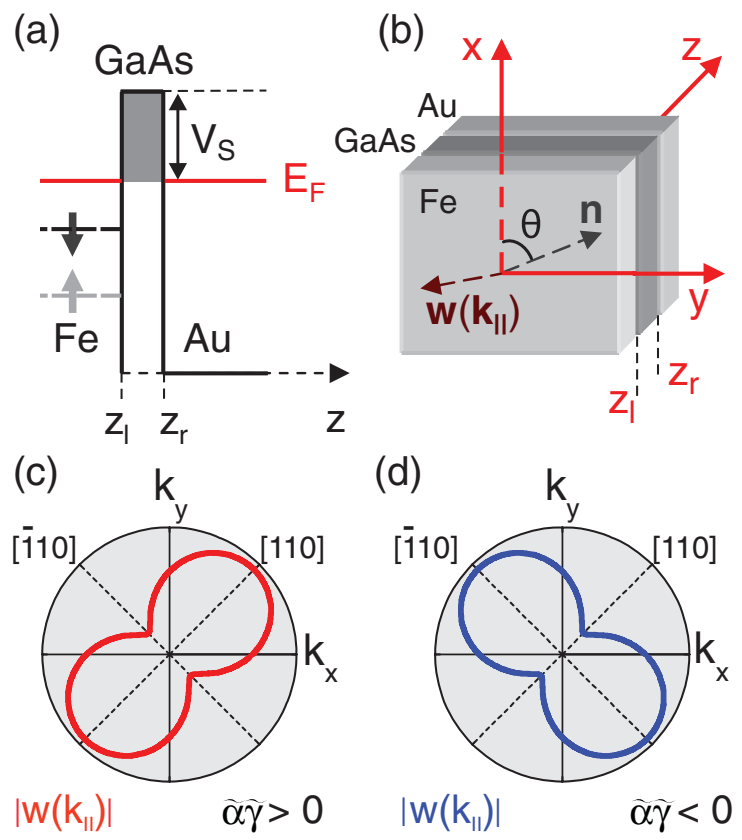

FIG. 3 (color). (a) Schematics of the conduction band profile along the growth direction of the heterostructure; the tunneling barrier with the Schottky barrier height $V_{S}$ is gray shaded. Spin splitting of the bands in Fe is indicated. (b) A spacial view of the model system. The vector $\mathbf{n}$ determines the magnetization direction in Fe with respect to the [100] direction ( $x$ axis). The SOI-induced spin precession of the electrons during tunneling is characterized by the vector $\mathbf{w}\left(\mathbf{k}_{\|}\right)$(see text). Polar plots of $\left|\mathbf{w}\left(\mathbf{k}_{\|}\right)\right|$for a fixed value of $\left|\mathbf{k}_{\|}\right|$are schematically represented in (c) and (d) for cases in which $\tilde{\alpha} \tilde{\gamma}>0$ and $\tilde{\alpha} \tilde{\gamma}<0$, respectively. The anisotropy of the field $\mathbf{w}\left(\mathbf{k}_{\|}\right)$in the $\mathbf{k}_{\|}$space is transferred to the tunneling transmissivity (see text) and transformed into the observed anisotropy of the TAMR.

The Dresselhaus SOI resulting from the bulk inversion asymmetry in GaAs is incorporated in the model through the term $[11,12,15-17]$

$$
H_{D}=\frac{1}{\hbar}\left(\sigma_{x} p_{x}-\sigma_{y} p_{y}\right) \frac{\partial}{\partial z}\left(\gamma(z) \frac{\partial}{\partial z}\right),
$$

where the Dresselhaus parameter $\gamma \approx 24 \mathrm{eV} \AA^{3}$ in the GaAs region [12,15-17] and $\gamma=0$ elsewhere.

The current flowing along the heterojunction is

$$
I=\frac{e}{(2 \pi)^{3} \hbar} \sum_{\sigma=-1,1} \int d E d^{2} k_{\|} T_{\sigma}\left(E, \mathbf{k}_{\|}\right)\left[f_{l}(E)-f_{r}(E)\right]
$$

where $\mathbf{k}_{\|}$is the in-plane wave vector and $f_{l}(E)$ and $f_{r}(E)$ are the electron Fermi-Dirac distributions with chemical potentials $\mu_{l}$ and $\mu_{r}$ in the left and right leads, respectively. The particle transmissivity $T_{\sigma}\left(E, \mathbf{k}_{\|}\right)$is found by solving for the scattering states in the different regions.

The calculations of the dependence of the resistance on the angle $\theta$ between $\mathbf{M}$ in Fe and the [100] direction (note that $\theta=\phi+\pi / 4$ ) were carried out at $T=0$ and a barrier height $V_{S}=0.75 \mathrm{mV}$. For the Fe layer, we assume a Stoner 
model with the majority and minority spin channels having Fermi momenta $k_{F \uparrow}=1.05 \times 10^{8} \mathrm{~cm}^{-1}$ and $k_{F \downarrow}=0.44 \times$ $10^{8} \mathrm{~cm}^{-1}$ [18], respectively. The Fermi momentum in $\mathrm{Au}$ was assumed to be $k_{F}=1.2 \times 10^{8} \mathrm{~cm}^{-1}$ [19].

The values of the Bychkov-Rashba parameters $\alpha_{l}, \alpha_{r}$ [see Eq. (3)] are not known for metal-semiconductor interfaces. Because of the complexity of the problem, a theoretical estimation of such parameters requires first principle calculations including the band structure details of the involved materials, which is beyond the scope of the present Letter. Here we assume $\alpha_{l}$ and $\alpha_{r}$ as phenomenological parameters. We have found that, due to the large exchange splitting in the left $(\mathrm{Fe})$ region, the calculated TAMR is dominated by $\alpha_{l}$; the dependence on $\alpha_{r}$ is negligible, and we can set $\alpha_{r}=0$. This leaves only $\alpha_{l}$ as a fitting parameter in the comparison of the theoretical and experimental value of the ratio $R_{[1 \overline{0} 0]} / R_{[110]}$. Such a comparison is displayed in Fig. 2(a) for the case of an external bias $V_{\text {bias }}=-90 \mathrm{meV}$ and low magnetic field $B=0.5 \mathrm{~T}$. The agreement between theory and experiment is indeed very satisfactory, considering that we fit the value of $\alpha_{l}$ (the fit is $42.3 \mathrm{eV} \AA^{2}$ ) only for the direction $\phi=\pi / 2$ - this is enough for our theoretical model to reproduce the complete angular dependence of $R(\phi) / R_{[110]}$. Preliminary ab initio calculations confirm qualitatively the above picture [20].

We have performed the same fitting procedure for other values of the applied voltage, shown in Figs. 2(b) and 2(c), with good agreement between theory and experiment. Different values of $\alpha_{l}$ are obtained when varying the bias, suggesting that the interface Bychkov-Rashba parameters are voltage-dependent (unlike $\gamma$, which is a material parameter), as found in other systems [1]. The interface Bychkov-Rashba parameter $\alpha_{l}$ in our system changes sign at a bias slightly below $50 \mathrm{mV}$.

The robustness of the fit points to the following phenomenological model of the TAMR. Averaging the SOI $H_{\mathrm{SOI}}=H_{\mathrm{BR}}+H_{D}$ [see Eqs. (3) and (4)] over the states of the system, one obtains $H_{\text {SOI }} \sim \mathbf{w}\left(\mathbf{k}_{\|}\right) \cdot \boldsymbol{\sigma}$ [16], where $\mathbf{w}\left(\mathbf{k}_{\|}\right)=\left(\tilde{\alpha} k_{y}-\tilde{\gamma} k_{x},-\tilde{\alpha} k_{x}+\tilde{\gamma} k_{y}, 0\right)$. Here $\tilde{\alpha}$ and $\tilde{\gamma}$ are effective Bychkov-Rashba and Dresselhaus parameters that measure the SOI-induced spin precession of the electrons during tunneling. There are only two preferential directions defined by $\mathbf{n}$ and $\mathbf{w}\left(\mathbf{k}_{\|}\right)$[see Fig. 3(b)]. Therefore, the anisotropy of a scalar quantity such as the total transmissivity is obtained as a perturbative series of $\mathbf{n} \cdot \mathbf{w}\left(\mathbf{k}_{\|}\right)$, since the SOI is much smaller than the other relevant energy scales in the system. Averaging over the inplane momenta to get the full current, the anisotropy is determined, up to the second order, by $\left\langle\left[\mathbf{n} \cdot \mathbf{w}\left(\mathbf{k}_{\|}\right)\right]^{2}\right\rangle$ [the first-order term vanishes, since $\left.\mathbf{w}\left(\mathbf{k}_{\|}\right)=-\mathbf{w}\left(-\mathbf{k}_{\|}\right)\right]$. Thus, the tunneling current anisotropy is proportional to $\tilde{\alpha} \tilde{\gamma} \sin 2 \theta$ [21]. Taking into account that $\theta=\phi+\pi / 4$ and that the observed anisotropy is small, one obtains for the TAMR $R(\phi) / R_{[110]}-1 \sim \tilde{\alpha} \tilde{\gamma}(\cos 2 \phi-1)$. This is precisely the kind of angular dependence experimentally found (see Fig. 2). Assuming that the spin-orbit parameters are voltage-dependent, one can change the sign and magnitude of the anisotropy $\tilde{\alpha} \tilde{\gamma}$ by varying the bias voltage, as shown in Fig. 2. Notably, if $\tilde{\alpha} \tilde{\gamma} \approx 0$, one obtains a suppression of the TAMR effect, a situation corresponding to a bias voltage of $50 \mathrm{mV}$ [Fig. 2(c)].

We thank M. Sperl for SQUID measurements and M. Lobenhofer for communicating TAMR data at $100 \mathrm{~K}$. Financial support by DFG via No. SFB 689 and by BMBF (nanoQUIT) is gratefully acknowledged.

Note added. - A recent ab initio calculation on $\mathrm{Fe}(001)$ surfaces [22] has demonstrated a large TAMR due to the Bychkov-Rashba spin splitting, supporting our interpretation in terms of the interface spin-orbit coupling.

[1] I. Žutić, J. Fabian, and S. Das Sarma, Rev. Mod. Phys. 76, 323 (2004).

[2] M. Jullière, Phys. Lett. 54, 225 (1975).

[3] C. Gould et al., Phys. Rev. Lett. 93, 117203 (2004).

[4] L. Brey, C. Tejedor, and J. Fernández-Rossier, Appl. Phys. Lett. 85, 1996 (2004).

[5] A.D. Giddings et al., Phys. Rev. Lett. 94, 127202 (2005).

[6] J. Moser et al., Appl. Phys. Lett. 89, 162106 (2006).

[7] As the same wafer was used to prepare TMR devices with $\mathrm{Fe}$ instead of $\mathrm{Au}$, the Co layer was needed to pin the iron layer (see Ref. [6]). While the Co affects the switching field, it plays no role for the effect discussed here.

[8] S. Kreuzer et al., Appl. Phys. Lett. 80, 4582 (2002).

[9] R. P. Cowburn et al., J. Appl. Phys. 78, 7210 (1995).

[10] G. Dresselhaus, Phys. Rev. 100, 580 (1955).

[11] U. Rössler and J. Kainz, Solid State Commun. 121, 313 (2002).

[12] R. Winkler, Springer Tracts in Modern Physics (Springer, Berlin, 2003), Vol. 191.

[13] The Zeeman energy in GaAs is much smaller than all of the other energy scales characterizing the system, and we can therefore neglect its effect.

[14] E. A. de Andrada e Silva, G. C. La Rocca, and F. Bassani, Phys. Rev. B 50, 8523 (1994); 55, 16293 (1997).

[15] V. I. Perel et al., Phys. Rev. B 67, 201304(R) (2003).

[16] S. D. Ganichev et al., Phys. Rev. Lett. 92, 256601 (2004).

[17] L. G. Wang, W. Yang, K. Chang, and K. S. Chan, Phys. Rev. B 72, 153314 (2005).

[18] J. Wang, D. Y. Xing, and H. B. Sun, J. Phys. Condens. Matter 15, 4841 (2003).

[19] N. W. Ashcroft and N.D. Mermin, Solid State Physics (Saunders College, Philadelphia, 1988).

[20] V. Popescu and H. Ebert (private communication).

[21] A. Matos-Abiague and J. Fabian (unpublished).

[22] A. N. Chantis, K. D. Belashchenko, E. Y. Tsymbal, and M. van Schilfgaarde, Phys. Rev. Lett. 98, 046601 (2007). 